\title{
A Comparison of Approaches to Large-Scale Data Analysis
}

\author{
Andrew Pavlo \\ Brown University \\ pavlo@cs.brown.edu
}

\author{
Erik Paulson \\ University of Wisconsin \\ epaulson@cs.wisc.edu
}

\author{
Alexander Rasin \\ Brown University \\ alexr@cs.brown.edu
}

\author{
Daniel J. Abadi \\ Yale University \\ dna@cs.yale.edu \\ David J. DeWitt \\ Microsoft Inc. \\ dewitt@microsoft.com
}

\author{
Samuel Madden \\ M.I.T. CSAIL
}

\author{
Michael Stonebraker \\ M.I.T. CSAIL \\ stonebraker@csail.mit.edu
}

\begin{abstract}
There is currently considerable enthusiasm around the MapReduce (MR) paradigm for large-scale data analysis [17]. Although the basic control flow of this framework has existed in parallel SQL database management systems (DBMS) for over 20 years, some have called MR a dramatically new computing model $[8,17]$. In this paper, we describe and compare both paradigms. Furthermore, we evaluate both kinds of systems in terms of performance and development complexity. To this end, we define a benchmark consisting of a collection of tasks that we have run on an open source version of MR as well as on two parallel DBMSs. For each task, we measure each system's performance for various degrees of parallelism on a cluster of 100 nodes. Our results reveal some interesting trade-offs. Although the process to load data into and tune the execution of parallel DBMSs took much longer than the MR system, the observed performance of these DBMSs was strikingly better. We speculate about the causes of the dramatic performance difference and consider implementation concepts that future systems should take from both kinds of architectures.
\end{abstract}

\section{Categories and Subject Descriptors}

H.2.4 [Database Management]: Systems—Parallel databases

\section{General Terms}

Database Applications, Use Cases, Database Programming

\section{INTRODUCTION}

Recently the trade press has been filled with news of the revolution of "cluster computing". This paradigm entails harnessing large numbers of (low-end) processors working in parallel to solve a computing problem. In effect, this suggests constructing a data center by lining up a large number of low-end servers instead of deploying a smaller set of high-end servers. With this rise of interest in clusters has come a proliferation of tools for programming them. One of the earliest and best known such tools in MapReduce (MR) [8]. MapReduce is attractive because it provides a simple

Permission to make digital or hard copies of all or part of this work for personal or classroom use is granted without fee provided that copies are not made or distributed for profit or commercial advantage and that copies bear this notice and the full citation on the first page. To copy otherwise, to republish, to post on servers or to redistribute to lists, requires prior specific permission and/or a fee.

SIGMOD'09, June 29-July 2, 2009, Providence, Rhode Island, USA.

Copyright 2009 ACM 978-1-60558-551-2/09/06 ...\$5.00. model through which users can express relatively sophisticated distributed programs, leading to significant interest in the educational community. For example, IBM and Google have announced plans to make a 1000 processor MapReduce cluster available to teach students distributed programming.

Given this interest in MapReduce, it is natural to ask "Why not use a parallel DBMS instead?" Parallel database systems (which all share a common architectural design) have been commercially available for nearly two decades, and there are now about a dozen in the marketplace, including Teradata, Aster Data, Netezza, DATAllegro (and therefore soon Microsoft SQL Server via Project Madison), Dataupia, Vertica, ParAccel, Neoview, Greenplum, DB2 (via the Database Partitioning Feature), and Oracle (via Exadata). They are robust, high performance computing platforms. Like MapReduce, they provide a high-level programming environment and parallelize readily. Though it may seem that MR and parallel databases target different audiences, it is in fact possible to write almost any parallel processing task as either a set of database queries (possibly using user defined functions and aggregates to filter and combine data) or a set of MapReduce jobs. Inspired by this question, our goal is to understand the differences between the MapReduce approach to performing large-scale data analysis and the approach taken by parallel database systems. The two classes of systems make different choices in several key areas. For example, all DBMSs require that data conform to a well-defined schema, whereas MR permits data to be in any arbitrary format. Other differences also include how each system provides indexing and compression optimizations, programming models, the way in which data is distributed, and query execution strategies.

The purpose of this paper is to consider these choices, and the trade-offs that they entail. We begin in Section 2 with a brief review of the two alternative classes of systems, followed by a discussion in Section 3 of the architectural trade-offs. Then, in Section 4 we present our benchmark consisting of a variety of tasks, one taken from the MR paper [8], and the rest a collection of more demanding tasks. In addition, we present the results of running the benchmark on a 100-node cluster to execute each task. We tested the publicly available open-source version of MapReduce, Hadoop [1], against two parallel SQL DBMSs, Vertica [3] and a second system from a major relational vendor. We also present results on the time each system took to load the test data and report informally on the procedures needed to set up and tune the software for each task.

In general, the SQL DBMSs were significantly faster and required less code to implement each task, but took longer to tune and load the data. Hence, we conclude with a discussion on the reasons for the differences between the approaches and provide suggestions on the best practices for any large-scale data analysis engine.

Some readers may feel that experiments conducted using 100 
nodes are not interesting or representative of real world data processing systems. We disagree with this conjecture on two points. First, as we demonstrate in Section 4, at 100 nodes the two parallel DBMSs range from a factor of 3.1 to 6.5 faster than MapReduce on a variety of analytic tasks. While MR may indeed be capable of scaling up to 1000 s of nodes, the superior efficiency of modern DBMSs alleviates the need to use such massive hardware on datasets in the range of 1-2PB (1000 nodes with 2TB of disk/node has a total disk capacity of $2 \mathrm{~PB}$ ). For example, eBay's Teradata configuration uses just 72 nodes (two quad-core CPUs, 32GB RAM, $104300 \mathrm{~GB}$ disks per node) to manage approximately $2.4 \mathrm{~PB}$ of relational data. As another example, Fox Interactive Media's warehouse is implemented using a 40-node Greenplum DBMS. Each node is a Sun X4500 machine with two dual-core CPUs, 48 500GB disks, and 16 GB RAM (1PB total disk space) [7]. Since few data sets in the world even approach a petabyte in size, it is not at all clear how many MR users really need 1,000 nodes.

\section{TWO APPROACHES TO LARGE SCALE DATA ANALYSIS}

The two classes of systems we consider in this paper run on a "shared nothing" collection of computers [19]. That is, the system is deployed on a collection of independent machines, each with local disk and local main memory, connected together on a highspeed local area network. Both systems achieve parallelism by dividing any data set to be utilized into partitions, which are allocated to different nodes to facilitate parallel processing. In this section, we provide an overview of how both the MR model and traditional parallel DBMSs operate in this environment.

\subsection{MapReduce}

One of the attractive qualities about the MapReduce programming model is its simplicity: an MR program consists only of two functions, called Map and Reduce, that are written by a user to process key/value data pairs. The input data set is stored in a collection of partitions in a distributed file system deployed on each node in the cluster. The program is then injected into a distributed processing framework and executed in a manner to be described.

The Map function reads a set of "records" from an input file, does any desired filtering and/or transformations, and then outputs a set of intermediate records in the form of new key/value pairs. As the Map function produces these output records, a "split" function partitions the records into $R$ disjoint buckets by applying a function to the key of each output record. This split function is typically a hash function, though any deterministic function will suffice. Each map bucket is written to the processing node's local disk. The Map function terminates having produced $R$ output files, one for each bucket. In general, there are multiple instances of the Map function running on different nodes of a compute cluster. We use the term instance to mean a unique running invocation of either the Map or Reduce function. Each Map instance is assigned a distinct portion of the input file by the MR scheduler to process. If there are $M$ such distinct portions of the input file, then there are $R$ files on disk storage for each of the $M$ Map tasks, for a total of $M \times R$ files; $F_{i j}, 1 \leq i \leq M, 1 \leq j \leq R$. The key observation is that all Map instances use the same hash function; thus, all output records with the same hash value are stored in the same output file.

The second phase of a MR program executes $R$ instances of the Reduce program, where $R$ is typically the number of nodes. The input for each Reduce instance $R_{j}$ consists of the files $F_{i j}, 1 \leq$ $i \leq M$. These files are transferred over the network from the Map nodes' local disks. Note that again all output records from the Map phase with the same hash value are consumed by the same Reduce instance, regardless of which Map instance produced the data. Each Reduce processes or combines the records assigned to it in some way, and then writes records to an output file (in the distributed file system), which forms part of the computation's final output.

The input data set exists as a collection of one or more partitions in the distributed file system. It is the job of the MR scheduler to decide how many Map instances to run and how to allocate them to available nodes. Likewise, the scheduler must also decide on the number and location of nodes running Reduce instances. The MR central controller is responsible for coordinating the system activities on each node. A MR program finishes execution once the final result is written as new files in the distributed file system.

\subsection{Parallel DBMSs}

Database systems capable of running on clusters of shared nothing nodes have existed since the late 1980s. These systems all support standard relational tables and SQL, and thus the fact that the data is stored on multiple machines is transparent to the end-user. Many of these systems build on the pioneering research from the Gamma [10] and Grace [11] parallel DBMS projects. The two key aspects that enable parallel execution are that (1) most (or even all) tables are partitioned over the nodes in a cluster and that (2) the system uses an optimizer that translates SQL commands into a query plan whose execution is divided amongst multiple nodes. Because programmers only need to specify their goal in a high level language, they are not burdened by the underlying storage details, such as indexing options and join strategies.

Consider a SQL command to filter the records in a table $T_{1}$ based on a predicate, along with a join to a second table $T_{2}$ with an aggregate computed on the result of the join. A basic sketch of how this command is processed in a parallel DBMS consists of three phases. Since the database will have already stored $T_{1}$ on some collection of the nodes partitioned on some attribute, the filter sub-query is first performed in parallel at these sites similar to the filtering performed in a Map function. Following this step, one of two common parallel join algorithms are employed based on the size of data tables. For example, if the number of records in $T_{2}$ is small, then the DBMS could replicate it on all nodes when the data is first loaded. This allows the join to execute in parallel at all nodes. Following this, each node then computes the aggregate using its portion of the answer to the join. A final "roll-up" step is required to compute the final answer from these partial aggregates [9].

If the size of the data in $T_{2}$ is large, then $T_{2}$ 's contents will be distributed across multiple nodes. If these tables are partitioned on different attributes than those used in the join, the system will have to hash both $T_{2}$ and the filtered version of $T_{1}$ on the join attribute using a common hash function. The redistribution of both $T_{2}$ and the filtered version of $T_{1}$ to the nodes is similar to the processing that occurs between the Map and the Reduce functions. Once each node has the necessary data, it then performs a hash join and calculates the preliminary aggregate function. Again, a roll-up computation must be performed as a last step to produce the final answer.

At first glance, these two approaches to data analysis and processing have many common elements; however, there are notable differences that we consider in the next section.

\section{ARCHITECTURAL ELEMENTS}

In this section, we consider aspects of the two system architectures that are necessary for processing large amounts of data in a distributed environment. One theme in our discussion is that the nature of the MR model is well suited for development environments with a small number of programmers and a limited application domain. This lack of constraints, however, may not be appropriate for longer-term and larger-sized projects. 


\subsection{Schema Support}

Parallel DBMSs require data to fit into the relational paradigm of rows and columns. In contrast, the MR model does not require that data files adhere to a schema defined using the relational data model. That is, the MR programmer is free to structure their data in any manner or even to have no structure at all.

One might think that the absence of a rigid schema automatically makes MR the preferable option. For example, SQL is often criticized for its requirement that the programmer must specify the "shape" of the data in a data definition facility. On the other hand, the MR programmer must often write a custom parser in order to derive the appropriate semantics for their input records, which is at least an equivalent amount of work. But there are also other potential problems with not using a schema for large data sets.

Whatever structure exists in MR input files must be built into the Map and Reduce programs. Existing MR implementations provide built-in functionality to handle simple key/value pair formats, but the programmer must explicitly write support for more complex data structures, such as compound keys. This is possibly an acceptable approach if a MR data set is not accessed by multiple applications. If such data sharing exists, however, a second programmer must decipher the code written by the first programmer to decide how to process the input file. A better approach, followed by all SQL DBMSs, is to separate the schema from the application and store it in a set of system catalogs that can be queried.

But even if the schema is separated from the application and made available to multiple MR programs through a description facility, the developers must also agree on a single schema. This obviously requires some commitment to a data model or models, and the input files must obey this commitment as it is cumbersome to modify data attributes once the files are created.

Once the programmers agree on the structure of data, something or someone must ensure that any data added or modified does not violate integrity or other high-level constraints (e.g., employee salaries must be non negative). Such conditions must be known and explicitly adhered to by all programmers modifying a particular data set; a MR framework and its underlying distributed storage system has no knowledge of these rules, and thus allows input data to be easily corrupted with bad data. By again separating such constraints from the application and enforcing them automatically by the run time system, as is done by all SQL DBMSs, the integrity of the data is enforced without additional work on the programmer's behalf.

In summary, when no sharing is anticipated, the MR paradigm is quite flexible. If sharing is needed, however, then we argue that it is advantageous for the programmer to use a data description language and factor schema definitions and integrity constraints out of application programs. This information should be installed in common system catalogs accessible to the appropriate users and applications.

\subsection{Indexing}

All modern DBMSs use hash or B-tree indexes to accelerate access to data. If one is looking for a subset of records (e.g., employees with a salary greater than $\$ 100,000$ ), then using a proper index reduces the scope of the search dramatically. Most database systems also support multiple indexes per table. Thus, the query optimizer can decide which index to use for each query or whether to simply perform a brute-force sequential search.

Because the MR model is so simple, MR frameworks do not provide built-in indexes. The programmer must implement any indexes that they may desire to speed up access to the data inside of their application. This is not easily accomplished, as the framework's data fetching mechanisms must also be instrumented to use these indexes when pushing data to running Map instances. Once more, this is an acceptable strategy if the indexes do not need to be shared between multiple programmers, despite requiring every MR programmer re-implement the same basic functionality.

If sharing is needed, however, then the specifications of what indexes are present and how to use them must be transferred between programmers. It is again preferable to store this index information in a standard format in the system catalogs, so that programmers can query this structure to discover such knowledge.

\subsection{Programming Model}

During the 1970s, the database research community engaged in a contentious debate between the relational advocates and the Codasyl advocates [18]. The salient issue of this discussion was whether a program to access data in a DBMS should be written either by:

1. Stating what you want - rather than presenting an algorithm for how to get it (Relational)

\section{Presenting an algorithm for data access (Codasyl)}

In the end, the former view prevailed and the last 30 years is a testament to the value of relational database systems. Programs in high-level languages, such as SQL, are easier to write, easier to modify, and easier for a new person to understand. Codasyl was criticized for being "the assembly language of DBMS access". We argue that MR programming is somewhat analogous to Codasyl programming: one is forced to write algorithms in a low-level language in order to perform record-level manipulation. On the other hand, to many people brought up programming in procedural languages, such as $\mathrm{C} / \mathrm{C}++$ or Java, describing tasks in a declarative language like SQL can be challenging.

Anecdotal evidence from the MR community suggests that there is widespread sharing of MR code fragments to do common tasks, such as joining data sets. To alleviate the burden of having to reimplement repetitive tasks, the MR community is migrating highlevel languages on top of the current interface to move such functionality into the run time. Pig [15] and Hive [2] are two notable projects in this direction.

\subsection{Data Distribution}

The conventional wisdom for large-scale databases is to always send the computation to the data, rather than the other way around. In other words, one should send a small program over the network to a node, rather than importing a large amount of data from the node. Parallel DBMSs use knowledge of data distribution and location to their advantage: a parallel query optimizer strives to balance computational workloads while minimizing the amount data transmitted over the network connecting the nodes of the cluster.

Aside from the initial decision on where to schedule Map instances, a MR programmer must perform these tasks manually. For example, suppose a user writes a MR program to process a collection of documents in two parts. First, the Map function scans the documents and creates a histogram of frequently occurring words. The documents are then passed to a Reduce function that groups files by their site of origin. Using this data, the user, or another user building on the first user's work, now wants to find sites with a document that contains more than five occurrences of the word 'Google' or the word 'IBM'. In the naive implementation of this query, where the Map is executed over the accumulated statistics, the filtration is done after the statistics for all documents are computed and shipped to reduce workers, even though only a small subset of documents satisfy the keyword filter.

In contrast, the following SQL view and select queries perform a similar computation: 
CREATE VIEW Keywords AS

SELECT siteid, docid, word, COUNT(*) AS wordcount

FROM Documents

GROUP BY siteid, docid, word,

SELECT DISTINCT siteid

FROM Keywords

WHERE (word $=$ 'IBM' OR word $=$ 'Google') AND wordcount $>5$;

A modern DBMS would rewrite the second query such that the view definition is substituted for the Keywords table in the FROM clause. Then, the optimizer can push the WHERE clause in the query down so that it is applied to the Documents table before the COUNT is computed, substantially reducing computation. If the documents are spread across multiple nodes, then this filter can be applied on each node before documents belonging to the same site are grouped together, generating much less network I/O.

\subsection{Execution Strategy}

There is a potentially serious performance problem related to MR's handling of data transfer between Map and Reduce jobs. Recall that each of the $N$ Map instances produces $M$ output files, each destined for a different Reduce instance. These files are written to the local disk on the node executing each particular Map instance. If $N$ is 1000 and $M$ is 500 , the Map phase of the program produces 500,000 local files. When the Reduce phase starts, each of the 500 Reduce instances needs to read its 1000 input files and must use a file-transfer protocol to "pull" each of its input files from the nodes on which the Map instances were run. With 100s of Reduce instances running simultaneously, it is inevitable that two or more Reduce instances will attempt to read their input files from the same map node simultaneously, inducing large numbers of disk seeks and slowing the effective disk transfer rate. This is why parallel database systems do not materialize their split files and instead use a push approach to transfer data instead of a pull.

\subsection{Flexibility}

Despite its widespread adoption, SQL is routinely criticized for its insufficient expressive prowess. Some believe that it was a mistake for the database research community in the 1970s to focus on data sub-languages that could be embedded in any programming language, rather than adding high-level data access to all programming languages. Fortunately, new application frameworks, such as Ruby on Rails [21] and LINQ [14], have started to reverse this situation by leveraging new programming language functionality to implement an object-relational mapping pattern. These programming environments allow developers to benefit from the robustness of DBMS technologies without the burden of writing complex SQL.

Proponents of the MR model argue that SQL does not facilitate the desired generality that MR provides. But almost all of the major DBMS products (commercial and open-source) now provide support for user-defined functions, stored procedures, and user-defined aggregates in SQL. Although this does not have the full generality of MR, it does improve the flexibility of database systems.

\subsection{Fault Tolerance}

The MR frameworks provide a more sophisticated failure model than parallel DBMSs. While both classes of systems use some form of replication to deal with disk failures, MR is far more adept at handling node failures during the execution of a MR computation. In a MR system, if a unit of work (i.e., processing a block of data) fails, then the MR scheduler can automatically restart the task on an alternate node. Part of the flexibility is the result of the fact that the output files of the Map phase are materialized locally instead of being streamed to the nodes running the Reduce tasks. Similarly, pipelines of MR jobs, such as the one described in Section 4.3.4, materialize intermediate results to files each step of the way. This differs from parallel DBMSs, which have larger granules of work (i.e., transactions) that are restarted in the event of a failure. Part of the reason for this approach is that DBMSs avoid saving intermediate results to disk whenever possible. Thus, if a single node fails during a long running query in a DBMS, the entire query must be completely restarted.

\section{PERFORMANCE BENCHMARKS}

In this section, we present our benchmark consisting of five tasks that we use to compare the performance of the MR model with that of parallel DBMSs. The first task is taken directly from the original MapReduce paper [8] that the authors' claim is representative of common MR tasks. Because this task is quite simple, we also developed four additional tasks, comprised of more complex analytical workloads designed to explore the trade-offs discussed in the previous section. We executed our benchmarks on a well-known MR implementation and two parallel DBMSs.

\subsection{Benchmark Environment}

As we describe the details of our benchmark environment, we note how the different data analysis systems that we test differ in operating assumptions and discuss the ways in which we dealt with them in order to make the experiments uniform.

\subsubsection{Tested Systems}

Hadoop: The Hadoop system is the most popular open-source implementation of the MapReduce framework, under development by Yahoo! and the Apache Software Foundation [1]. Unlike the Google implementation of the original MR framework written in $\mathrm{C}++$, the core Hadoop system is written entirely in Java. For our experiments in this paper, we use Hadoop version 0.19.0 running on Java 1.6.0. We deployed the system with the default configuration settings, except for the following changes that we found yielded better performance without diverging from core MR fundamentals: (1) data is stored using $256 \mathrm{MB}$ data blocks instead of the default $64 \mathrm{MB},(2)$ each task executor JVM ran with a maximum heap size of 512MB and the DataNode/JobTracker JVMs ran with a maximum heap size of a $1024 \mathrm{MB}$ (for a total size of $3.5 \mathrm{~GB}$ per node), (3) we enabled Hadoop's "rack awareness" feature for data locality in the cluster, and (4) we allowed Hadoop to reuse the task JVM executor instead starting a new process for each Map/Reduce task. Moreover, we configured the system to run two Map instances and a single Reduce instance concurrently on each node.

The Hadoop framework also provides an implementation of the Google distributed file system [12]. For each benchmark trial, we store all input and output data in the Hadoop distributed file system (HDFS). We used the default settings of HDFS of three replicas per block and without compression; we also tested other configurations, such as using only a single replica per block as well as blockand record-level compression, but we found that our tests almost always executed at the same speed or worse with these features enabled (see Section 5.1.3). After each benchmark run finishes for a particular node scaling level, we delete the data directories on each node and reformat HDFS so that the next set of input data is replicated uniformly across all nodes.

Hadoop uses a central job tracker and a "master" HDFS daemon to coordinate node activities. To ensure that these daemons do not affect the performance of worker nodes, we execute both of these additional framework components on a separate node in the cluster.

DBMS-X: We used the latest release of DBMS-X, a parallel SQL DBMS from a major relational database vendor that stores data in 
a row-based format. The system is installed on each node and configured to use 4GB shared memory segments for the buffer pool and other temporary space. Each table is hash partitioned across all nodes on the salient attribute for that particular table, and then sorted and indexed on different attributes (see Sections 4.2.1 and 4.3.1). Like the Hadoop experiments, we deleted the tables in DBMS$\mathrm{X}$ and reloaded the data for each trial to ensure that the tuples was uniformly distributed in the cluster.

By default DBMS-X does not compress data in its internal storage, but it does provide ability to compress tables using a wellknown dictionary-based scheme. We found that enabling compression reduced the execution times for almost all the benchmark tasks by $50 \%$, and thus we only report results with compression enabled. In only one case did we find that using compression actually performed worse. Furthermore, because all of our benchmarks are read-only, we did not enable replication features in DBMS-X, since this would not have improved performance and complicates the installation process.

Vertica: The Vertica database is a parallel DBMS designed for large data warehouses [3]. The main distinction of Vertica from other DBMSs (including DBMS-X) is that all data is stored as columns, rather than rows [20]. It uses a unique execution engine designed specifically for operating on top of a column-oriented storage layer. Unlike DBMS-X, Vertica compresses data by default since its executor can operate directly on compressed tables. Because disabling this feature is not typical in Vertica deployments, the Vertica results in this paper are generated using only compressed data. Vertica also sorts every table by one or more attributes based on a clustered index.

We found that the default $256 \mathrm{MB}$ buffer size per node performed well in our experiments. The Vertica resource manager is responsible for setting the amount of memory given to queries, but we provide a hint to the system to expect to execute only one query at a time. Thus, each query receives most the maximum amount of memory available on each node at runtime.

\subsubsection{Node Configuration}

All three systems were deployed on a 100-node cluster. Each node has a single $2.40 \mathrm{GHz}$ Intel Core 2 Duo processor running 64bit Red Hat Enterprise Linux 5 (kernel version 2.6.18) with 4GB RAM and two 250GB SATA-I hard disks. According to hdparm, the hard disks deliver $7 \mathrm{~GB} / \mathrm{sec}$ for cached reads and about $74 \mathrm{MB} / \mathrm{sec}$ for buffered reads. The nodes are connected with Cisco Catalyst 3750E-48TD switches. This switch has gigabit Ethernet ports for each node and an internal switching fabric of 128Gbps [6]. There are 50 nodes per switch. The switches are linked together via Cisco StackWise Plus, which creates a 64Gbps ring between the switches. Traffic between two nodes on the same switch is entirely local to the switch and does not impact traffic on the ring.

\subsubsection{Benchmark Execution}

For each benchmark task, we describe the steps used to implement the MR program as well as provide the equivalent SQL statement(s) executed by the two database systems. We executed each task three times and report the average of the trials. Each system executes the benchmark tasks separately to ensure exclusive access to the cluster's resources. To measure the basic performance without the overhead of coordinating parallel tasks, we first execute each task on a single node. We then execute the task on different cluster sizes to show how each system scales as both the amount of data processed and available resources are increased. We only report results using trials where all nodes are available and the system's software operates correctly during the benchmark execution.
We also measured the time it takes for each system to load the test data. The results from these measurements are split between the actual loading of the data and any additional operations after the loading that each system performs, such as compressing or building indexes. The initial input data on each node is stored on one of its two locally installed disks.

Unless otherwise indicated, the final results from the queries executing in Vertica and DBMS-X are piped from a shell command into a file on the disk not used by the DBMS. Although it is possible to do an equivalent operation in Hadoop, it is easier (and more common) to store the results of a MR program into the distributed file system. This procedure, however, is not analogous to how the DBMSs produce their output data; rather than storing the results in a single file, the MR program produces one output file for each Reduce instance and stores them in a single directory. The standard practice is for developers then to use these output directories as a single input unit for other MR jobs. If, however, a user wishes to use this data in a non-MR application, they must first combine the results into a single file and download it to the local file system.

Because of this discrepancy, we execute an extra Reduce function for each MR benchmark task that simply combines the final output into a single file in HDFS. Our results differentiate between the execution times for Hadoop running the actual benchmark task versus the additional combine operation. Thus, the Hadoop results displayed in the graphs for this paper are shown as stacked bars: the lower portion of each bar is the execution time for just the specific benchmark task, while the upper portion is the execution time for the single Reduce function to combine all of the program's output data into a single file.

\subsection{The Original MR Task}

Our first benchmark task is the "Grep task" taken from the original MapReduce paper, which the authors describe as "representative of a large subset of the real programs written by users of MapReduce" [8]. For this task, each system must scan through a data set of 100-byte records looking for a three-character pattern. Each record consists of a unique key in the first 10 bytes, followed by a 90 -byte random value. The search pattern is only found in the last 90 bytes once in every 10,000 records.

The input data is stored on each node in plain text files, with one record per line. For the Hadoop trials, we uploaded these files unaltered directly into HDFS. To load the data into Vertica and DBMS$\mathrm{X}$, we execute each system's proprietary load commands in parallel on each node and store the data using the following schema:

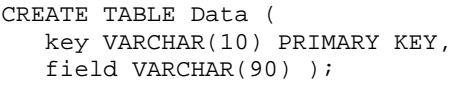

We execute the Grep task using two different data sets. The measurements in the original MapReduce paper are based on processing $1 \mathrm{~TB}$ of data on approximately 1800 nodes, which is 5.6 million records or roughly $535 \mathrm{MB}$ of data per node. For each system, we execute the Grep task on cluster sizes of $1,10,25,50$, and 100 nodes. The total number of records processed for each cluster size is therefore 5.6 million times the number of nodes. The performance of each system not only illustrates how each system scales as the amount of data is increased, but also allows us to (to some extent) compare the results to the original MR system.

While our first dataset fixes the size of the data per node to be the same as the original MR benchmark and only varies the number of nodes, our second dataset fixes the total dataset size to be the same as the original MR benchmark (1TB) and evenly divides the data amongst a variable number of nodes. This task measures how well each system scales as the number of available nodes is increased. 


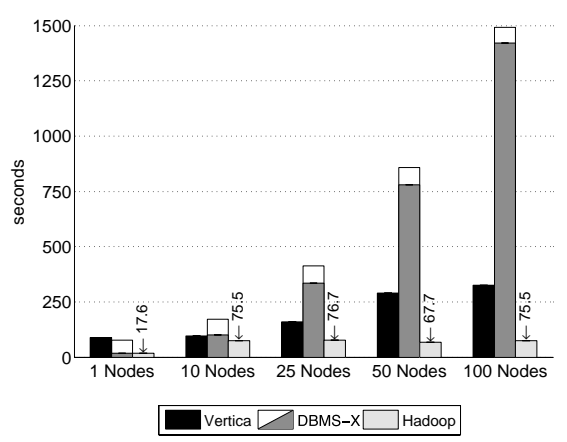

1: Load Times - Grep Task Data Set (535MB/node)

Since Hadoop needs a total of 3TB of disk space in order to store three replicas of each block in HDFS, we were limited to running this benchmark only on 25, 50, and 100 nodes (at fewer than 25 nodes, there is not enough available disk space to store 3TB).

\subsubsection{Data Loading}

We now describe the procedures used to load the data from the nodes' local files into each system's internal storage representation.

Hadoop: There are two ways to load data into Hadoop's distributed file system: (1) use Hadoop's command-line file utility to upload files stored on the local filesystem into HDFS or (2) create a custom data loader program that writes data using Hadoop's internal I/O API. We did not need to alter the input data for our MR programs, therefore we loaded the files on each node in parallel directly into HDFS as plain text using the command-line utility. Storing the data in this manner enables MR programs to access data using Hadoop's Text InputFormat data format, where the keys are line numbers in each file and their corresponding values are the contents of each line. We found that this approach yielded the best performance in both the loading process and task execution, as opposed to using Hadoop's serialized data formats or compression features.

DBMS-X: The loading process in DBMS-X occurs in two phases. First, we execute the LOAD SQL command in parallel on each node in the cluster to read data from the local filesystem and insert its contents into a particular table in the database. We specify in this command that the local data is delimited by a special character, thus we did not need to write a custom program to transform the data before loading it. But because our data generator simply creates random keys for each record on each node, the system must redistribute the tuples to other nodes in the cluster as it reads each record from the input files based on the target table's partitioning attribute. It would be possible to generate a "hash-aware" version of the data generator that would allow DBMS-X to just load the input files on each node without this redistribution process, but we do not believe that this would improve load times very much.

Once the initial loading phase is complete, we then execute an administrative command to reorganize the data on each node. This process executes in parallel on each node to compress data, build each table's indexes, and perform other housekeeping.

Vertica: Vertica also provides a COPY SQL command that is issued from a single host and then coordinates the loading process on multiple nodes in parallel in the cluster. The user gives the COPY command as input a list of nodes to execute the loading operation for. This process is similar to DBMS-X: on each node the Vertica loader splits the input data files on a delimiter, creates a new tuple for each line in an input file, and redistributes that tuple to a dif-

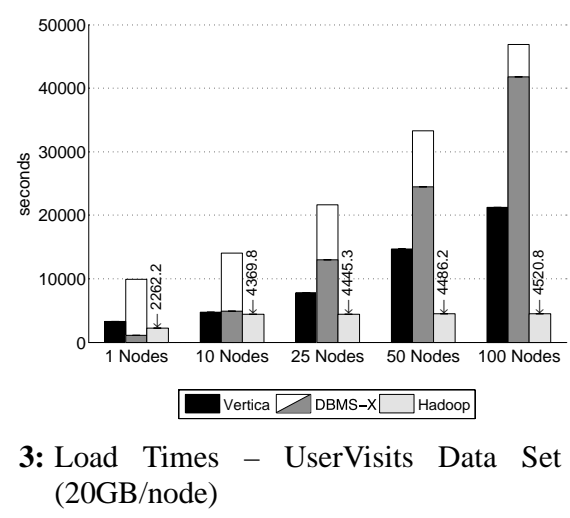

ferent node based on the hash of its primary key. Once the data is loaded, the columns are automatically sorted and compressed according to the physical design of the database.

Results \& Discussion: The results for loading both the $535 \mathrm{MB} /$ node and 1TB/cluster data sets are shown in Figures 1 and 2, respectively. For DBMS-X, we separate the times of the two loading phases, which are shown as a stacked bar in the graphs: the bottom segment represents the execution time of the parallel LOAD commands and the top segment is the reorganization process.

The most striking feature of the results for the load times in $535 \mathrm{MB} /$ node data set shown in Figure 1 is the difference in performance of DBMS-X compared to Hadoop and Vertica. Despite issuing the initial LOAD command in the first phase on each node in parallel, the data was actually loaded on each node sequentially. Thus, as the total of amount of data is increased, the load times also increased proportionately. This also explains why, for the $1 \mathrm{~TB} / \mathrm{cluster}$ data set, the load times for DBMS-X do not decrease as less data is stored per node. However, the compression and housekeeping on DBMS-X can be done in parallel across nodes, and thus the execution time of the second phase of the loading process is cut in half when twice as many nodes are used to store the 1TB of data.

Without using either block- or record-level compression, Hadoop clearly outperforms both DBMS-X and Vertica since each node is simply copying each data file from the local disk into the local HDFS instance and then distributing two replicas to other nodes in the cluster. If we load the data into Hadoop using only a single replica per block, then the load times are reduced by a factor of three. But as we will discuss in Section 5, the lack of multiple replicas often increases the execution times of jobs.

\subsubsection{Task Execution}

SQL Commands: A pattern search for a particular field is simply the following query in SQL. Neither SQL system contained an index on the field attribute, so this query requires a full table scan.

SELECT * FROM Data WHERE field LIKE 'XYZ\%';

MapReduce Program: The MR program consists of just a Map function that is given a single record already split into the appropriate key/value pair and then performs a sub-string match on the value. If the search pattern is found, the Map function simply outputs the input key/value pair to HDFS. Because no Reduce function is defined, the output generated by each Map instance is the final output of the program.

Results \& Discussion: The performance results for the three systems for this task is shown in Figures 4 and 5. Surprisingly, the relative differences between the systems are not consistent in the two figures. In Figure 4, the two parallel databases perform about 


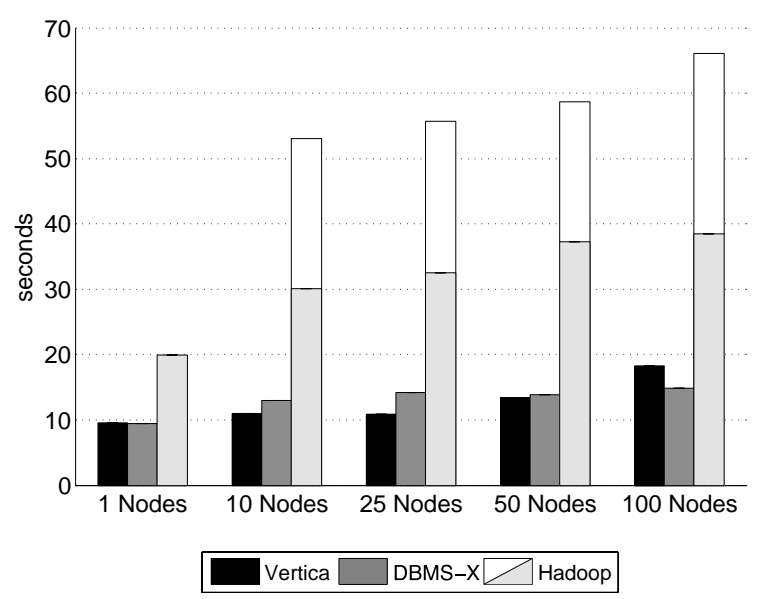

4: Grep Task Results - 535MB/node Data Set

the same, more than a factor of two faster in Hadoop. But in Figure 5, both DBMS-X and Hadoop perform more than a factor of two slower than Vertica. The reason is that the amount of data processing varies substantially from the two experiments. For the results in Figure 4, very little data is being processed (535MB/node). This causes Hadoop's non-insignificant start-up costs to become the limiting factor in its performance. As will be described in Section 5.1.2, for short-running queries (i.e., queries that take less than a minute), Hadoop's start-up costs can dominate the execution time. In our observations, we found that takes 10-25 seconds before all Map tasks have been started and are running at full speed across the nodes in the cluster. Furthermore, as the total number of allocated Map tasks increases, there is additional overhead required for the central job tracker to coordinate node activities. Hence, this fixed overhead increases slightly as more nodes are added to the cluster and for longer data processing tasks, as shown in Figure 5, this fixed cost is dwarfed by the time to complete the required processing.

The upper segments of each Hadoop bar in the graphs represent the execution time of the additional MR job to combine the output into a single file. Since we ran this as a separate MapReduce job, these segments consume a larger percentage of overall time in Figure 4, as the fixed start-up overhead cost again dominates the work needed to perform the rest of the task. Even though the Grep task is selective, the results in Figure 5 show how this combine phase can still take hundreds of seconds due to the need to open and combine many small output files. Each Map instance produces its output in a separate HDFS file, and thus even though each file is small there are many Map tasks and therefore many files on each node.

For the 1TB/cluster data set experiments, Figure 5 shows that all systems executed the task on twice as many nodes in nearly half the amount of time, as one would expect since the total amount of data was held constant across nodes for this experiment. Hadoop and DBMS-X performs approximately the same, since Hadoop's startup cost is amortized across the increased amount of data processing for this experiment. However, the results clearly show that Vertica outperforms both DBMS-X and Hadoop. We attribute this to Vertica's aggressive use of data compression (see Section 5.1.3), which becomes more effective as more data is stored per node.

\subsection{Analytical Tasks}

To explore more complex uses of both types of systems, we developed four tasks related to HTML document processing. We first generate a collection of random HTML documents, similar to that which a web crawler might find. Each node is assigned a set of 600,000 unique HTML documents, each with a unique URL. In

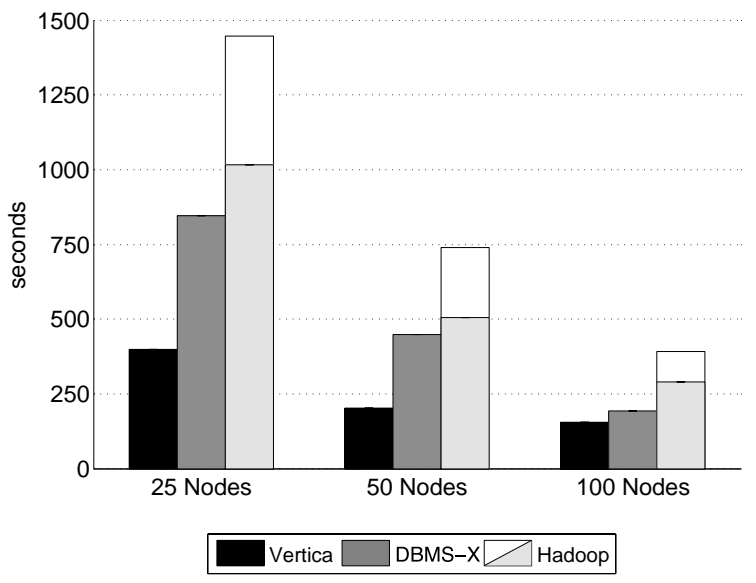

5: Grep Task Results - 1TB/cluster Data Set

each document, we randomly generate links to other pages set using a Zipfian distribution.

We also generated two additional data sets meant to model log files of HTTP server traffic. These data sets consist of values derived from the HTML documents as well as several randomly generated attributes. The schema of these three tables is as follows:

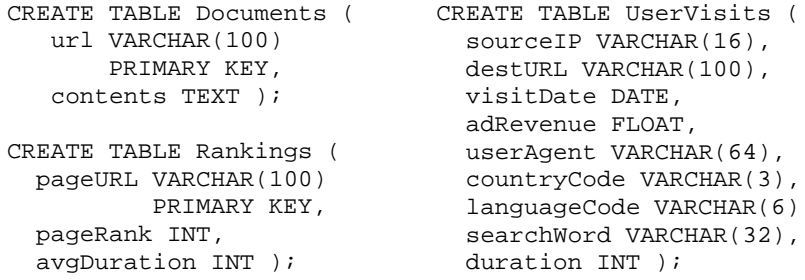

Our data generator created unique files with 155 million UserVisits records (20GB/node) and 18 million Rankings records (1GB/node) on each node. The visitDate, adRevenue, and sourceIP fields are picked uniformly at random from specific ranges. All other fields are picked uniformly from sampling real-world data sets. Each data file is stored on each node as a column-delimited text file.

\subsubsection{Data Loading}

We now describe the procedures for loading the UserVisits and Rankings data sets. For reasons to be discussed in Section 4.3.5, only Hadoop needs to directly load the Documents files into its internal storage system. DBMS-X and Vertica both execute a UDF that processes the Documents on each node at runtime and loads the data into a temporary table. We account for the overhead of this approach in the benchmark times, rather than in the load times. Therefore, we do not provide results for loading this data set.

Hadoop: Unlike the Grep task's data set, which was uploaded directly into HDFS unaltered, the UserVisits and Rankings data sets needed to be modified so that the first and second columns are separated by a tab delimiter and all other fields in each line are separated by a unique field delimiter. Because there are no schemas in the MR model, in order to access the different attributes at run time, the Map and Reduce functions in each task must manually split the value by the delimiter character into an array of strings.

We wrote a custom data loader executed in parallel on each node to read in each line of the data sets, prepare the data as needed, and then write the tuple into a plain text file in HDFS. Loading the data sets in this manner was roughly three times slower than using the command-line utility, but did not require us to write custom input handlers in Hadoop; the MR programs are able to use 


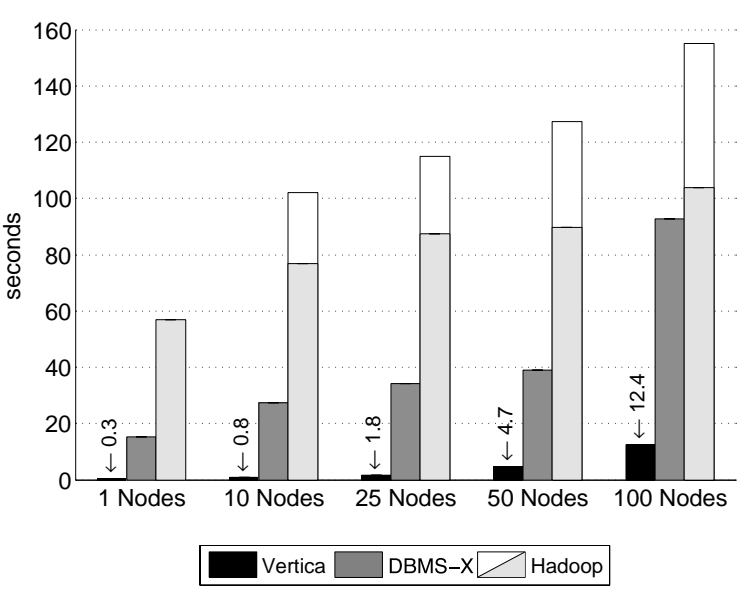

6: Selection Task Results

Hadoop's KeyValueText InputFormat interface on the data files to automatically split lines of text files into key/values pairs by the tab delimiter. Again, we found that other data format options, such as SequenceFileInputFormat or custom Writable tuples, resulted in both slower load and execution times.

DBMS-X: We used the same loading procedures for DBMS-X as discussed in Section 4.2. The Rankings table was hash partitioned across the cluster on pageURL and the data on each node was sorted by pageRank. Likewise, the UserVisits table was hash partitioned on destinationURL and sorted by visitDate on each node.

Vertica: Similar to DBMS-X, Vertica used the same bulk load commands discussed in Section 4.2 and sorted the UserVisits and Rankings tables by the visitDate and pageRank columns, respectively.

Results \& Discussion: Since the results of loading the UserVisits and Ranking data sets are similar, we only provide the results for loading the larger UserVisits data in Figure 3. Just as with loading the Grep 535MB/node data set (Figure 1), the loading times for each system increases in proportion to the number of nodes used.

\subsubsection{Selection Task}

The Selection task is a lightweight filter to find the pageURLs in the Rankings table (1GB/node) with a pageRank above a userdefined threshold. For our experiments, we set this threshold parameter to 10 , which yields approximately 36,000 records per data file on each node.

SQL Commands: The DBMSs execute the selection task using the following simple SQL statement:

SELECT pageURL, pageRank

FROM Rankings WHERE pageRank $>\mathrm{X}_{\text {; }}$

MapReduce Program: The MR program uses only a single Map function that splits the input value based on the field delimiter and outputs the record's pageURL and pageRank as a new key/value pair if its pageRank is above the threshold. This task does not require a Reduce function, since each pageURL in the Rankings data set is unique across all nodes.

Results \& Discussion: As was discussed in the Grep task, the results from this experiment, shown in Figure 6, demonstrate again that the parallel DBMSs outperform Hadoop by a rather significant factor across all cluster scaling levels. Although the relative performance of all systems degrade as both the number of nodes and the total amount of data increase, Hadoop is most affected. For example, there is almost a $50 \%$ difference in the execution time between the 1 node and 10 node experiments. This is again due to Hadoop's increased start-up costs as more nodes are added to the cluster, which takes up a proportionately larger fraction of total query time for short-running queries.

Another important reason for why the parallel DBMSs are able to outperform Hadoop is that both Vertica and DBMS-X use an index on the pageRank column and store the Rankings table already sorted by pageRank. Thus, executing this query is trivial. It should also be noted that although Vertica's absolute times remain low, its relative performance degrades as the number of nodes increases. This is in spite of the fact that each node still executes the query in the same amount of time (about $170 \mathrm{~ms}$ ). But because the nodes finish executing the query so quickly, the system becomes flooded with control messages from too many nodes, which then takes a longer time for the system to process. Vertica uses a reliable message layer for query dissemination and commit protocol processing [4], which we believe has considerable overhead when more than a few dozen nodes are involved in the query.

\subsubsection{Aggregation Task}

Our next task requires each system to calculate the total adRevenue generated for each sourceIP in the UserVisits table (20GB/node), grouped by the sourceIP column. We also ran a variant of this query where we grouped by the seven-character prefix of the sourceIP column to measure the effect of reducing the total number of groups on query performance. We designed this task to measure the performance of parallel analytics on a single read-only table, where nodes need to exchange intermediate data with one another in order compute the final value. Regardless of the number of nodes in the cluster, this tasks always produces 2.5 million records $(53 \mathrm{MB})$; the variant query produces 2,000 records $(24 \mathrm{~KB})$.

SQL Commands: The SQL commands to calculate the total adRevenue is straightforward:

SELECT SourCeIP, SUM(adRevenue)

FROM UserVisits GROUP BY sourceIP;

The variant query is:

SELECT SUBSTR (sourceIP, 1, 7), SUM (adRevenue)

FROM UserVisits GROUP BY SUBSTR(sourceIP, 1, 7);

MapReduce Program: Unlike the previous tasks, the MR program for this task consists of both a Map and Reduce function. The Map function first splits the input value by the field delimiter, and then outputs the sourceIP field (given as the input key) and the adRevenue field as a new key/value pair. For the variant query, only the first seven characters (representing the first two octets, each stored as three digits) of the sourceIP are used. These two Map functions share the same Reduce function that simply adds together all of the adRevenue values for each sourceIP and then outputs the prefix and revenue total. We also used MR's Combine feature to perform the pre-aggregate before data is transmitted to the Reduce instances, improving the first query's execution time by a factor of two [8].

Results \& Discussion: The results of the aggregation task experiment in Figures 7 and 8 show once again that the two DBMSs outperform Hadoop. The DBMSs execute these queries by having each node scan its local table, extract the sourceIP and adRevenue fields, and perform a local group by. These local groups are then merged at the query coordinator, which outputs results to the user. The results in Figure 7 illustrate that the two DBMSs perform about the same 


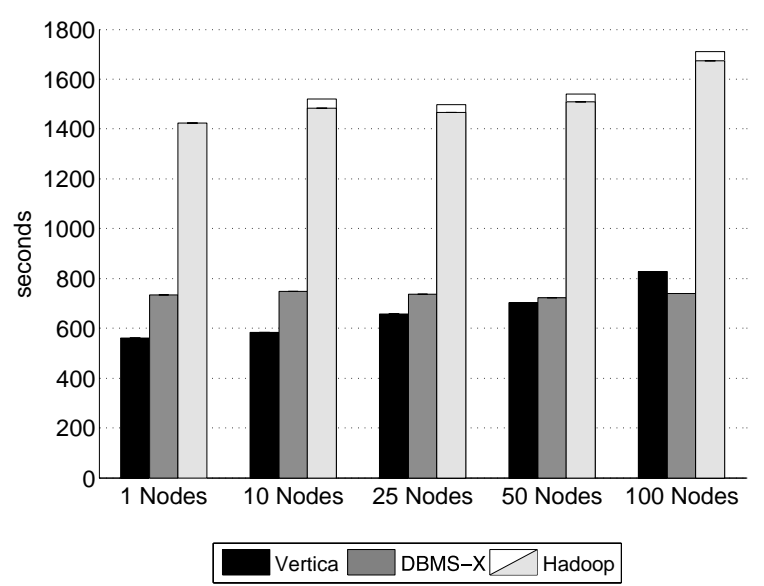

7: Aggregation Task Results (2.5 million Groups)

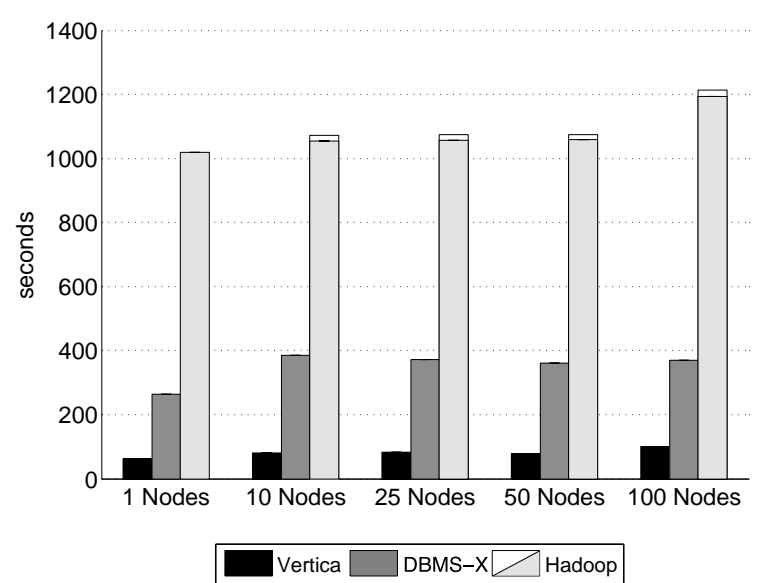

8: Aggregation Task Results (2,000 Groups)

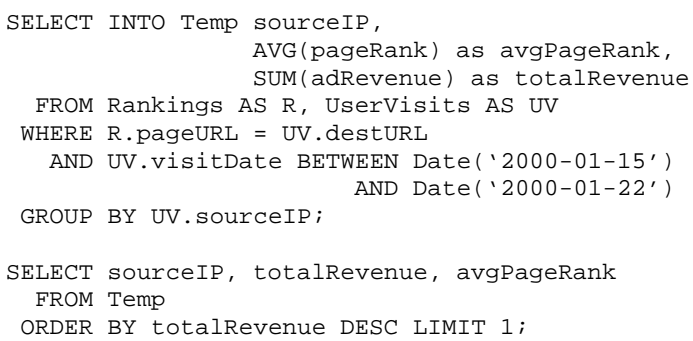

MapReduce Program: Because the MR model does not have an inherent ability to join two or more disparate data sets, the MR program that implements the join task must be broken out into three separate phases. Each of these phases is implemented together as a single MR program in Hadoop, but do not begin executing until the previous phase is complete.

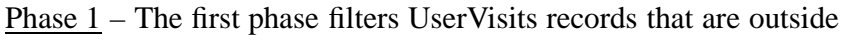
the desired data range and then joins the qualifying records with records from the Rankings file. The MR program is initially given all of the UserVisits and Rankings data files as input.

Map Function: For each key/value input pair, we determine its record type by counting the number of fields produced when splitting the value on the delimiter. If it is a UserVisits record, we apply the filter based on the date range predicate. These qualifying records are emitted with composite keys of the form (destURL, $K_{1}$ ), where $K_{1}$ indicates that it is a UserVisits record. All Rankings records are emitted with composite keys of the form (pageURL, $K_{2}$ ), where $K_{2}$ indicates that it is a Rankings record. These output records are repartitioned using a user-supplied partitioning function that only hashes on the URL portion of the composite key.

Reduce Function: The input to the Reduce function is a single sorted run of records in URL order. For each URL, we divide its values into two sets based on the tag component of the composite key. The function then forms the cross product of the two sets to complete the join and outputs a new key/value pair with the sourceIP as the key and the tuple (pageURL, pageRank, adRevenue) as the value.

Phase 2 - The next phase computes the total adRevenue and average pageRank based on the sourceIP of records generated in Phase 1. This phase uses a Reduce function in order to gather all of the records for a particular sourceIP on a single node. We use the identity Map function in the Hadoop API to supply records directly to the split process $[1,8]$. 


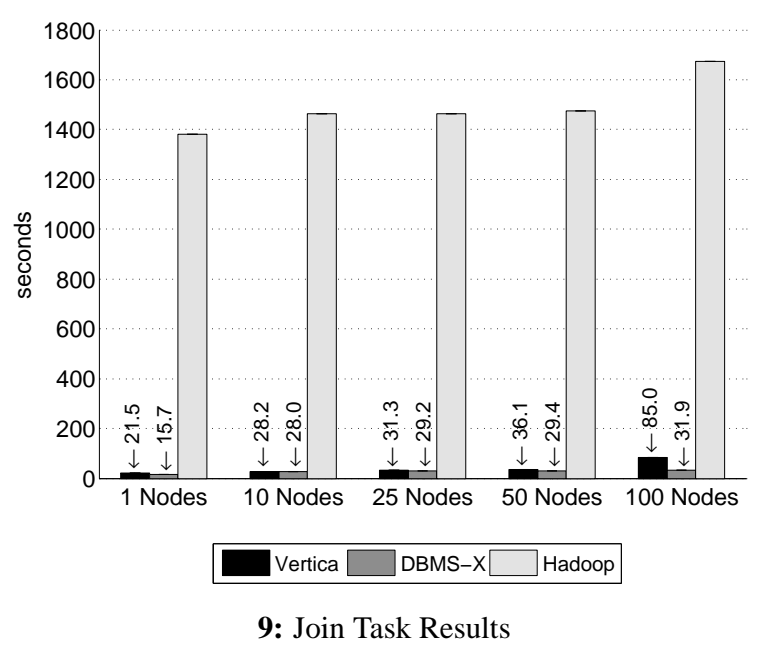

Reduce Function: For each sourceIP, the function adds up the adRevenue and computes the average pageRank, retaining the one with the maximum total ad revenue. Each Reduce instance outputs a single record with sourceIP as the key and the value as a tuple of the form (avgPageRank, totalRevenue).

$\underline{\text { Phase } 3}$ - In the final phase, we again only need to define a single Reduce function that uses the output from the previous phase to produce the record with the largest total adRevenue. We only execute one instance of the Reduce function on a single node to scan all the records from Phase 2 and find the target record.

Reduce Function: The function processes each key/value pair and keeps track of the record with the largest totalRevenue field. Because the Hadoop API does not easily expose the total number records that a Reduce instance will process, there is no way for the Reduce function to know that it is processing the last record. Therefore, we override the closing callback method in our Reduce implementation so that the MR program outputs the largest record right before it exits.

Results \& Discussion: The performance results for this task is displayed in Figure 9. We had to slightly change the SQL used in 100 node experiments for Vertica due to an optimizer bug in the system, which is why there is an increase in the execution time for Vertica going from 50 to 100 nodes. But even with this increase, it is clear that this task results in the biggest performance difference between Hadoop and the parallel database systems. The reason for this disparity is two-fold.

First, despite the increased complexity of the query, the performance of Hadoop is yet again limited by the speed with which the large UserVisits table (20GB/node) can be read off disk. The MR program has to perform a complete table scan, while the parallel database systems were able to take advantage of clustered indexes on UserVisits.visitDate to significantly reduce the amount of data that needed to be read. When breaking down the costs of the different parts of the Hadoop query, we found that regardless of the number of nodes in the cluster, phase 2 and phase 3 took on average 24.3 seconds and 12.7 seconds, respectively. In contrast, phase 1, which contains the Map task that reads in the UserVisits and Rankings tables, takes an average of 1434.7 seconds to complete. Interestingly, it takes approximately 600 seconds of raw I/O to read the UserVisits and Rankings tables off of disk and then another 300 seconds to split, parse, and deserialize the various attributes. Thus, the CPU overhead needed to parse these tables on the fly is the limiting factor for Hadoop.

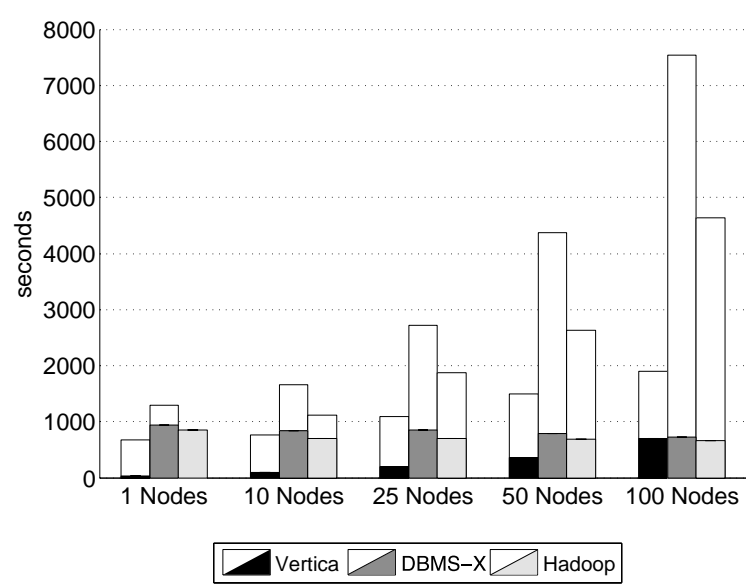

10: UDF Aggregation Task Results

Second, the parallel DBMSs are able to take advantage of the fact that both the UserVisits and the Rankings tables are partitioned by the join key. This means that both systems are able to do the join locally on each node, without any network overhead of repartitioning before the join. Thus, they simply have to do a local hash join between the Rankings table and a selective part of the UserVisits table on each node, with a trivial ORDER BY clause across nodes.

\subsubsection{UDF Aggregation Task}

The final task is to compute the inlink count for each document in the dataset, a task that is often used as a component of PageRank calculations. Specifically, for this task, the systems must read each document file and search for all the URLs that appear in the contents. The systems must then, for each unique URL, count the number of unique pages that reference that particular URL across the entire set of files. It is this type of task that the MR is believed to be commonly used for.

We make two adjustments for this task in order to make processing easier in Hadoop. First, we allow the aggregate to include self-references, as it is non-trivial for a Map function to discover the name of the input file it is processing. Second, on each node we concatenate the HTML documents into larger files when storing them in HDFS. We found this improved Hadoop's performance by a factor of two and helped avoid memory issues with the central HDFS master when a large number of files are stored in the system.

SQL Commands: To perform this task in a parallel DBMS requires a user-defined function $F$ that parses the contents of each record in the Documents table and emits URLs into the database. This function can be written in a general-purpose language and is effectively identical to the Map program discussed below. With this function $F$, we populate a temporary table with a list of URLs and then can execute a simple query to calculate the inlink count:

SELECT INTO Temp F(contents) FROM Documents;

SELECT url, SUM(value) FROM Temp GROUP BY url;

Despite the simplicity of this proposed UDF, we found that in practice it was difficult to implement in the DBMSs.

For DBMS-X, we translated the MR program used in Hadoop into an equivalent $\mathrm{C}$ program that uses the POSIX regular expression library to search for links in the document. For each URL found in the document contents, the UDF returns a new tuple (URL, 1) to the database engine. We originally intended to store each HTML document as a character BLOB in DBMS-X and then execute the UDF on each document completely inside of the database, 
but were unable to do so due to a known bug in our version of the system. Instead, we modified the UDF to open each HTML document on the local disk and process its contents as if it was stored in the database. Although this is similar to the approach that we had to take with Vertica (see below), the DBMS-X UDF did not run as an external process to the database and did not require any bulk-loading tools to import the extracted URLs.

Vertica does not currently support UDFs, therefore we had to implement this benchmark task in two phases. In the first phase, we used a modified version of DBMS-X's UDF to extract URLs from the files, but then write the output to files on each node's local filesystem. Unlike DBMS-X, this program executes as a separate process outside of the database system. Each node then loads the contents of these files into a table using Vertica's bulk-loading tools. Once this is completed, we then execute the query as described above to compute the inlink count for each URL.

MapReduce Program: To fit into the MR model where all data must be defined in terms of key/value pairs, each HTML document is split by its lines and given to the Map function with the line contents as the value and the line number in which it appeared in the file as its key. The Map function then uses a regular expression to find all of the URLs in each line. For every URL found, the function outputs the URL and the integer 1 as a new key/value pair. Given these records, the Reduce function then simply counts the number of values for a given key and outputs the URL and the calculated inlink count as the program's final output.

Results \& Discussion: The results in Figure 10 show that both DBMS-X and Hadoop (not including the extra Reduce process to combine the data) have approximately constant performance for this task, since each node has the same amount of Document data to process and this amount of data remains constant $(7 \mathrm{~GB})$ as more nodes are added in the experiments. As we expected, the additional operation for Hadoop to combine data into a single file in HDFS gets progressively slower since the amount of output data that the single node must process gets larger as new nodes are added. The results for both DBMS-X and Vertica are shown in Figure 10 as stacked bars, where the bottom segment represents the time it took to execute the UDF/parser and load the data into the table and the top segment is the time to execute the actual query. DBMS-X performs worse than Hadoop due to the added overhead of row-by-row interaction between the UDF and the input file stored outside of the database. Vertica's poor performance is the result of having to parse data outside of the DBMS and materialize the intermediate results on the local disk before it can load it into the system.

\section{DISCUSSION}

We now discuss broader issues about the benchmark results and comment on particular aspects of each system that the raw numbers may not convey. In the benchmark above, both DBMS-X and Vertica execute most of the tasks much faster than Hadoop at all scaling levels. The next subsections describe, in greater detail than the previous section, the reasons for this dramatic performance difference.

\subsection{System-level Aspects}

In this section, we describe how architectural decisions made at the system-level affect the relative performance of the two classes of data analysis systems. Since installation and configuration parameters can have a significant difference in the ultimate performance of the system, we begin with a discussion of the relative ease with which these parameters are set. Afterwards, we discuss some lower level implementation details. While some of these details affect performance in fundamental ways (e.g., the fact that MR does not transform data on loading precludes various I/O optimizations and necessitates runtime parsing which increases CPU costs), others are more implementation specific (e.g., the high start-up cost of MR).

\subsubsection{System Installation, Configuration, and Tuning}

We were able to get Hadoop installed and running jobs with little effort. Installing the system only requires setting up data directories on each node and deploying the system library and configuration files. Configuring the system for optimal performance was done through trial and error. We found that certain parameters, such as the size of the sort buffers or the number of replicas, had no affect on execution performance, whereas other parameters, such as using larger block sizes, improved performance significantly.

The DBMS-X installation process was relatively straightforward. A GUI leads the user through the initial steps on one of the cluster nodes, and then prepares a file that can be fed to an installer utility in parallel on the other nodes to complete the installation. Despite this simple process, we found that DBMS-X was complicated to configure in order to start running queries. Initially, we were frustrated by the failure of anything but the most basic of operations. We eventually discovered each node's kernel was configured to limit the total amount of allocated virtual address space. When this limit was hit, new processes could not be created and DBMS-X operations would fail. We mention this even though it was our own administrative error, as we were surprised that DBMS-X's extensive system probing and self-adjusting configuration was not able to detect this limitation. This was disappointing after our earlier Hadoop successes.

Even after these earlier issues were resolved and we had DBMS$\mathrm{X}$ running, we were routinely stymied by other memory limitations. We found that certain default parameters, such as the sizes of the buffer pool and sort heaps, were too conservative for modern systems. Furthermore, DBMS-X proved to be ineffective at adjusting memory allocations for changing conditions. For example, the system automatically expanded our buffer pool from the default $4 \mathrm{MB}$ to only $5 \mathrm{MB}$ (we later forced it to $512 \mathrm{MB}$ ). It also warned us that performance could be degraded when we increased our sort heap size to $128 \mathrm{MB}$ (in fact, performance improved by a factor of 12). Manually changing some options resulted in the system automatically altering others. On occasion, this combination of manual and automatic changes resulted in a configuration for DBMS-X that caused it to refuse to boot the next time the system started. As most configuration settings required DBMS-X to be running in order to adjust them, it was unfortunately easy to lock ourselves out with no failsafe mode to restore to a previous state.

Vertica was relatively easy to install as an RPM that we deployed on each node. An additional configuration script bundled with the RPM is used to build catalog meta-data and modify certain kernel parameters. Database tuning is minimal and is done through hints to the resource manager; we found that the default settings worked well for us. The downside of this simplified tuning approach, however, is that there is no explicit mechanism to determine what resources were granted to a query nor is there a way to manually adjust per query resource allocation.

The take-away from our efforts is that we found parallel DBMSs to be much more challenging than Hadoop to install and configure properly. There is, however, a significant variation with respect to ease of installation and configuration across the different parallel database products. One small advantage for the database systems is that the tuning that is needed is mostly done prior to query execution, and that certain tuning parameters (e.g., sort buffer sizes) are suitable for all tasks. In contrast, for Hadoop we not only had to tune the system (e.g., block sizes), but we also occasionally needed to tune each individual task to work well with the system (e.g., changing code). Finally, the parallel database products came with 
tools to aid in the tuning process whereas with Hadoop we were forced to resort to trial and error tuning; clearly a more mature MR implementation could include such tuning tools as well.

\subsubsection{Task Start-up}

We found that our MR programs took some time before all nodes were running at full capacity. On a cluster of 100 nodes, it takes 10 seconds from the moment that a job is submitted to the JobTracker before the first Map task begins to execute and 25 seconds until all the nodes in the cluster are executing the job. This coincides with the results in [8], where the data processing rate does not reach its peak for nearly 60 seconds on a cluster of 1800 nodes. The "cold start" nature is symptomatic to Hadoop's (and apparently Google's) implementation and not inherent to the actual MR model itself. For example, we also found that prior versions of Hadoop would create a new JVM process for each Map and Reduce instance on a node, which we found increased the overhead of running jobs on large data sets; enabling the JVM reuse feature in the latest version of Hadoop improved our results for MR by $10-15 \%$.

In contrast, parallel DBMSs are started at OS boot time, and thus are considered to always be "warm", waiting for a query to execute. Moreover, all modern DBMSs are designed to execute using multiple threads and processes, which allows the currently running code to accept additional tasks and further optimize its execution schedule. Minimizing start-up time was one of the early optimizations of DBMSs, and is certainly something that MR systems should be able to incorporate without a large rewrite of the underlying architecture.

\subsubsection{Compression}

Almost every parallel DBMS (including DBMS-X and Vertica) allows for optional compression of stored data. It is not uncommon for compression to result in a factor of 6-10 space savings. Vertica's internal data representation is highly optimized for data compression and has an execution engine that operates directly on compressed data (i.e., it avoids decompressing the data during processing whenever possible). In general, since analysis tasks on large data sets are often I/O bound, trading CPU cycles (needed to decompress input data) for $\mathrm{I} / \mathrm{O}$ bandwidth (compressed data means that there is less data to read) is a good strategy and translates to faster execution. In situations where the executor can operate directly on compressed data, there is often no trade-off at all and compression is an obvious win.

Hadoop and its underlying distributed filesystem support both block-level and record-level compression on input data. We found, however, that neither technique improved Hadoop's performance and in some cases actually slowed execution. It also required more effort on our part to either change code or prepare the input data. It should also be noted that compression was also not used in the original MR benchmark [8].

In order to use block-level compression in Hadoop, we first had to split the data files into multiple, smaller files on each node's local file system and then compress each file using the gzip tool. Compressing the data in this manner reduced each data set by $20-25 \%$ from its original size. These compressed files are then copied into HDFS just as if they were plain text files. Hadoop automatically detects when files are compressed and will decompress them on the fly when they are fed into Map instances, thus we did not need to change our MR programs to use the compressed data. Despite the longer load times (if one includes the splitting and compressing), Hadoop using block-level compression slowed most the tasks by a few seconds while CPU-bound tasks executed 50\% slower.

We also tried executing the benchmarks using record-level compression. This required us to (1) write to a custom tuple object using Hadoop's API, (2) modify our data loader program to transform records to compressed and serialized custom tuples, and (3) refactor each benchmark. We initially believed that this would improve CPU-bound tasks, because the Map and Reduce tasks no longer needed to split the fields by the delimiter. We found, however, that this approach actually performed worse than block-level compression while only compressing the data by $10 \%$.

\subsubsection{Loading and Data Layout}

Parallel DBMSs have the opportunity to reorganize the input data file at load time. This allows for certain optimizations, such as storing each attribute of a table separately (as done in column-stores such as Vertica). For read-only queries that only touch a subset of the attributes of a table, this optimization can improve performance by allowing the attributes that are not accessed by a particular query to be left on disk and never read. Similar to the compression optimization described above, this saves critical I/O bandwidth. MR systems by default do not transform the data when it is loaded into their distributed file system, and thus are unable to change the layout of input data, which precludes this class of optimization opportunities. Furthermore, Hadoop was always much more CPU intensive than the parallel DBMS in running equivalent tasks because it must parse and deserialize the records in the input data at run time, whereas parallel databases do the parsing at load time and can quickly extract attributes from tuples at essentially zero cost.

But MR's simplified loading process did make it much easier and faster to load than with the DBMSs. Our results in Sections 4.2.1 and 4.3.1 show that Hadoop achieved load throughputs of up to three times faster than Vertica and almost 20 times faster than DBMS-X. This suggests that for data that is only going to be loaded once for certain types on analysis tasks, that it may not be worth it to pay the cost of the indexing and reorganization cost in a DBMS . This also strongly suggests that a DBMS would benefit from a "insitu" operation mode that would allow a user to directly access and query files stored in a local file system.

\subsubsection{Execution Strategies}

As noted earlier, the query planner in parallel DBMSs are careful to transfer data between nodes only if it is absolutely necessary. This allows the systems to optimize the join algorithm depending on the characteristics of the data and perform push-oriented messaging without writing intermediate data sets. Over time, MR advocates should study the techniques used in parallel DBMSs and incorporate the concepts that are germane to their model. In doing so, we believe that again the performance of MR frameworks will improve dramatically.

Furthermore, parallel DBMSs construct a complete query plan that is sent to all processing nodes at the start of the query. Because data is "pushed" between sites when only necessary, there are no control messages during processing. In contrast, MR systems use a large number of control messages to synchronize processing, resulting in poorer performance due to increased overhead; Vertica also experienced this problem but on a much smaller scale (Section 4.2).

\subsubsection{Failure Model}

As discussed previously, while not providing support for transactions, MR is able to recover from faults in the middle of query execution in a way that most parallel database systems cannot. Since parallel DBMSs will be deployed on larger clusters over time, the probability of mid-query hardware failures will increase. Thus, for long running queries, it may be important to implement such a fault tolerance model. While improving the fault-tolerance of DBMSs is clearly a good idea, we are wary of devoting huge computational clusters and "brute force" approaches to computation when sophisticated software would could do the same processing with far less 
hardware and consume far less energy, or in less time, thereby obviating the need for a sophisticated fault tolerance model. A multithousand-node cluster of the sort Google, Microsoft, and Yahoo! run uses huge amounts of energy, and as our results show, for many data processing tasks a parallel DBMS can often achieve the same performance using far fewer nodes. As such, the desirable approach is to use high-performance algorithms with modest parallelism rather than brute force approaches on much larger clusters.

\subsection{User-level Aspects}

A data processing system's performance is irrelevant to a user or an organization if the system is not usable. In this section, we discuss aspects of each system that we encountered from a userlevel perspective while conducting the benchmark study that may promote or inhibit application development and adoption.

\subsubsection{Ease of Use}

Once the system is on-line and the data has been loaded, the programmer then begins to write the query or the code needed to perform their task. Like other kinds of programming, this is often an iterative process: the programmer writes a little bit of code, tests it, and then writes some more. The programmer can easily determine whether his/her code is syntactically correct in both types of systems: the MR framework can check whether the user's code compiles and the SQL engines can determine whether the queries parse correctly. Both systems also provide runtime support to assist users in debugging their programs.

It is also worth considering the way in which the programmer writes the query. MR programs in Hadoop are primarily written in Java (though other language bindings exist). Most programmers are more familiar with object-oriented, imperative programming than with other language technologies, such as SQL. That said, SQL is taught in many undergraduate programs and is fairly portable we were able to share the SQL commands between DBMS-X and Vertica with only minor modifications.

In general, we found that getting an MR program up and running with Hadoop took less effort than with the other systems. We did not need to construct a schema or register user-defined functions in order to begin processing the data. However, after obtaining our initial results, we expanded the number of benchmark tasks, causing us to add new columns to our data set. In order to process this new data, we had to modify our existing MR code and retest each MR program to ensure that it worked with the new assumptions about the data's schema. Furthermore, some API methods in Hadoop were deprecated after we upgraded to newer versions of the system, which again required us to rewrite portions of our programs. In contrast, once we had built our initial SQL-based applications, we did not have to modify the code despite several changes to our benchmark schema.

We argue that although it may be easier to for developers to get started with MR, maintenance of MR programs is likely to lead to significant pain for applications developers over time. As we also argued in Section 3.1, reusing MR code between two deployments or on two different data sets is difficult, as there is no explicit representation of the schema for data used in the MR model.

\subsubsection{Additional Tools}

Hadoop comes with a rudimentary web interface that allows the user to browse the contents of the distributed filesystem and monitor the execution of jobs. Any additional tools would most likely at this time have to be developed in house.

SQL databases, on the other hand, have tons of existing tools and applications for reporting and data analysis. Entire software industries have developed around providing DBMS users with third-party extensions. The types of software that many of these tools include (1) data visualization, (2) business intelligence, (3) data mining, (4) data replication, and (5) automatic database design. Because MR technologies are still nascent, the market for such software for MR is limited; however, as the user base grows, many of the existing SQL-based tools will likely support MR systems.

\section{CONCLUSION}

There are a number of interesting conclusions that can be drawn from the results presented in this paper. First, at the scale of the experiments we conducted, both parallel database systems displayed a significant performance advantage over Hadoop MR in executing a variety of data intensive analysis benchmarks. Averaged across all five tasks at 100 nodes, DBMS-X was 3.2 times faster than MR and Vertica was 2.3 times faster than DBMS-X. While we cannot verify this claim, we believe that the systems would have the same relative performance on 1,000 nodes (the largest Teradata configuration is less than 100 nodes managing over four petabytes of data). The dual of these numbers is that a parallel database system that provides the same response time with far fewer processors will certainly uses far less energy; the MapReduce model on multi-thousand node clusters is a brute force solution that wastes vast amounts of energy. While it is rumored that the Google version of MR is faster than the Hadoop version, we did not have access to this code and hence could not test it. We are doubtful again, however, that there would be a substantial difference in the performance of the two versions as MR is always forced to start a query with a scan of the entire input file.

This performance advantage that the two database systems share is the result of a number of technologies developed over the past 25 years, including (1) B-tree indices to speed the execution of selection operations, (2) novel storage mechanisms (e.g., columnorientation), (3) aggressive compression techniques with ability to operate directly on compressed data, and (4) sophisticated parallel algorithms for querying large amounts of relational data. In the case of a column-store database like Vertica, only those columns that are needed to execute a query are actually read from disk. Furthermore, the column-wise storage of data results in better compression factors (approximately a factor of 2.0 for Vertica, versus a factor of 1.8 for DBMS-X and 1.25 for Hadoop); this also further reduces the amount of disk I/O that is performed to execute a query.

Although we were not surprised by the relative performance advantages provided by the two parallel database systems, we were impressed by how easy Hadoop was to set up and use in comparison to the databases. The Vertica installation process was also straightforward but temperamental to certain system parameters. DBMS$\mathrm{X}$, on the other hand, was difficult to configure properly and required repeated assistance from the vendor to obtain a configuration that performed well. For a mature product such as DBMS-X, the entire experience was indeed disappointing. Given the upfront cost advantage that Hadoop has, we now understand why it has quickly attracted such a large user community.

Extensibility was another area where we found the database systems we tested lacking. Extending a DBMS with user-defined types and functions is an idea that is now 25 years old [16]. Neither of the parallel systems we tested did a good job on the UDF aggregation tasks, forcing us to find workarounds when we encountered limitations (e.g., Vertica) and bugs (e.g., DBMS-X).

While all DB systems are tolerant of a wide variety of software failures, there is no question that MR does a superior job of minimizing the amount of work that is lost when a hardware failure occurs. This capability, however, comes with a potentially large performance penalty, due to the cost of materializing the intermediate files between the map and reduce phases. Left unanswered is how significant this performance penalty is. Unfortunately, to investi- 
gate this question properly requires implementing both the materialization and no-materialization strategies in a common framework, which is an effort beyond the scope of this paper. Despite a clear advantage in this domain, it is not completely clear how significant a factor Hadoop's ability to tolerate failures during execution really is in practice. In addition, if a MR system needs 1,000 nodes to match the performance of a 100 node parallel database system, it is ten times more likely that a node will fail while a query is executing. That said, better tolerance to failures is a capability that any database user would appreciate.

Many people find SQL difficult to use initially. This is partially due to having to think differently when solving a problem and that SQL has evolved into a complex language that is quite different than the original design by Don Chamberlin in the 1970s. Though most languages become more complex over time, SQL is particularly bad as many of its features were designed by competing database companies who each sought to include their own proprietary extensions.

Despite its faults, SQL is still a powerful tool. Consider the following query to generate a list of Employees ordered by their salaries and the corresponding rank of each salary (i.e., the highest paid employee gets a rank of one):

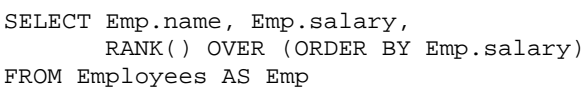

Computing this in parallel requires producing a total order of all employees followed by a second phase in which each node adjusts the rank values of its records with the counts of the number of records on each node to its "left" (i.e., those nodes with salary values that are strictly smaller). Although a MR program could perform this sort in parallel, it is not easy to fit this query into the MR paradigm of group by aggregation. RANK is just one of the many powerful analytic functions provided by modern parallel database systems. For example, both Teradata and Oracle support a rich set of functions, such as functions over windows of ordered records.

Two architectural differences are likely to remain in the long run. MR makes a commitment to a "schema later" or even "schema never" paradigm. But this lack of a schema has a number of important consequences. Foremost, it means that parsing records at run time is inevitable, in contrast to DBMSs, which perform parsing at load time. This difference makes compression less valuable in MR and causes a portion of the performance difference between the two classes of systems. Without a schema, each user must write a custom parser, complicating sharing data among multiple applications. Second, a schema is needed for maintaining information that is critical for optimizing declarative queries, including what indices exist, how tables are partitioned, table cardinalities, and histograms that capture the distribution of values within a column.

In our opinion there is a lot to learn from both kinds of systems. Most importantly is that higher level interfaces, such as Pig [15], Hive [2], are being put on top of the MR foundation, and a number of tools similar in spirit but more expressive than MR are being developed, such as Dryad [13] and Scope [5]. This will make complex tasks easier to code in MR-style systems and remove one of the big advantages of SQL engines, namely that they take much less code on the tasks in our benchmark. For parallel databases, we believe that both commercial and open-source systems will dramatically improve the parallelization of user-defined functions. Hence, the APIs of the two classes of systems are clearly moving toward each other. Early evidence of this is seen in the solutions for integrating SQL with MR offered by Greenplum and Asterdata.
The authors would like to thank Lakshmikant Shrinivas in helping to get DBMS-X running, as well as Chris Olston and the reviewers for their insightful comments and feedback. This work was supported in part by NSF Grant CluE - 0844013/0844480.

\section{REFERENCES}

[1] Hadoop. http://hadoop.apache.org/.

[2] Hive. http://hadoop.apache.org/hive/.

[3] Vertica. http://www.vertica.com/.

[4] Y. Amir and J. Stanton. The Spread Wide Area Group Communication System. Technical report, 1998.

[5] R. Chaiken, B. Jenkins, P.-A. Larson, B. Ramsey, D. Shakib, S. Weaver, and J. Zhou. Scope: easy and efficient parallel processing of massive data sets. Proc. VLDB Endow., 1(2):1265-1276, 2008.

[6] Cisco Systems. Cisco Catalyst 3750-E Series Switches Data Sheet, June 2008.

[7] J. Cohen, B. Dolan, M. Dunlap, J. M. Hellerstein, and C. Welton. MAD Skills: New Analysis Practices for Big Data. Under Submission, March 2009.

[8] J. Dean and S. Ghemawat. MapReduce: Simplified Data Processing on Large Clusters. In OSDI '04, pages 10-10, 2004.

[9] D. J. DeWitt and R. H. Gerber. Multiprocessor Hash-based Join Algorithms. In VLDB '85, pages 151-164, 1985.

[10] D. J. DeWitt, R. H. Gerber, G. Graefe, M. L. Heytens, K. B. Kumar, and M. Muralikrishna. GAMMA - A High Performance Dataflow Database Machine. In $V L D B$ '86, pages 228-237, 1986.

[11] S. Fushimi, M. Kitsuregawa, and H. Tanaka. An Overview of The System Software of A Parallel Relational Database Machine. In $V L D B$ '86, pages 209-219, 1986.

[12] S. Ghemawat, H. Gobioff, and S.-T. Leung. The Google File System. SIGOPS Oper. Syst. Rev., 37(5):29-43, 2003.

[13] M. Isard, M. Budiu, Y. Yu, A. Birrell, and D. Fetterly. Dryad: Distributed Data-parallel Programs from Sequential Building Blocks. In EuroSys '07, pages 59-72, 2007.

[14] E. Meijer, B. Beckman, and G. Bierman. LINQ: reconciling object, relations and XML in the .NET framework. In SIGMOD '06, pages 706-706, 2006.

[15] C. Olston, B. Reed, U. Srivastava, R. Kumar, and A. Tomkins. Pig Latin: A Not-So-Foreign Language for Data Processing. In SIGMOD '08, pages 1099-1110, 2008.

[16] J. Ong, D. Fogg, and M. Stonebraker. Implementation of data abstraction in the relational database system ingres. SIGMOD Rec., 14(1):1-14, 1983.

[17] D. A. Patterson. Technical Perspective: The Data Center is the Computer. Commun. ACM, 51(1):105-105, 2008.

[18] R. Rustin, editor. ACM-SIGMOD Workshop on Data Description, Access and Control, May 1974.

[19] M. Stonebraker. The Case for Shared Nothing. Database Engineering, 9:4-9, 1986.

[20] M. Stonebraker and J. Hellerstein. What Goes Around Comes Around. In Readings in Database Systems, pages 2-41. The MIT Press, 4th edition, 2005.

[21] D. Thomas, D. Hansson, L. Breedt, M. Clark, J. D. Davidson, J. Gehtland, and A. Schwarz. Agile Web Development with Rails. Pragmatic Bookshelf, 2006.

\section{ACKNOWLEDGMENTS}

